\title{
Behavioral Traits of the Online Parent-Child Game Players: A Case Study and Its Inspirations
}

\author{
Sujing Zhang and Feng Li \\ College of Education, Zhejiang Normal University, Jinhua, China \\ sjzhang@china.com, lifeng_nb@163.com
}

\begin{abstract}
By examining the online parent-child game entitled Island Survival, this paper probes into the behavioral traits of parent-child players by means of case study. The research mainly focuses on the following three points: (1). What kind of behaviors do the players conduct in the Online Parent-Child Game? (2). How many types of family relationship can be concluded on the basis of analyzing the players' behavioral traits? (3). With different types of family relationship sampled, check whether those types of family relationship are in accordance with the degrees of family intimacy based on the adaptive scales(FACESII-CV) or not. In the experiment, we choose students of two classes in a junior high school, as our samples, totaling 60 pairs of parent-child who are invited to experience a kind of parent-child game for 8 weeks, accumulating the storable and renewable database by means of snap-shooting and the temporarily deposited database in the server in the operation of games by a passive information-driven and an active rule-driven accumulation, and analyzing the dynamic data from the players' behaviors; We also choose four pairs of typical parent-child players. By further analyzing the social relationships of the families taking part in the online game, the time spanning of the parent-child players, and the branch task success ratio of parent role in parental education, the parent- kid family are classified into three different types: harmonious type, constructive type and crisis type, according to the players' behavioral traits; more importantly, the way of classifying offers critical enlightenment to the design of online parent-child games.
\end{abstract}

Keywords: Parent-Child Games, Parent-Child Cooperation, Player's Behavioral Traits, Data Analysis. 